\title{
O.S.P.
}

L'orientation scolaire et professionnelle

$30 / 3 \mid 2001$

Juger et être jugé : perspectives psychosociales

\section{Effet de contexte, valeur d'internalité et jugement scolaire}

Contextual effect, value of internality and school assessment

Pascal Bressoux et Pascal Pansu

\section{OpenEdition}

Journals

Édition électronique

URL : http://journals.openedition.org/osp/5133

DOI : $10.4000 /$ osp.5133

ISSN : 2104-3795

Éditeur

Institut national d'étude du travail et d'orientation professionnelle (INETOP)

Référence électronique

Pascal Bressoux et Pascal Pansu, «Effet de contexte, valeur d'internalité et jugement scolaire », L'orientation scolaire et professionnelle [En ligne], 30/3 | 2001, mis en ligne le 15 septembre 2004, consulté le 16 décembre 2020. URL : http://journals.openedition.org/osp/5133; DOI : https://doi.org/ $10.4000 /$ osp.5133

Ce document a été généré automatiquement le 16 décembre 2020.

(c) Tous droits réservés 


\title{
Effet de contexte, valeur d'internalité et jugement scolaire
}

\author{
Contextual effect, value of internality and school assessment
}

Pascal Bressoux et Pascal Pansu

\section{Introduction}

La présente étude s'inscrit dans le cadre d'un programme de recherche sur la construction du jugement scolaire des enseignants. Il ne s'agit pas ici d'apporter un regard docimologique (Pieron, 1963), mais de comprendre comment l'enseignant accomplit cette activité particulière, quels sont les mécanismes qui dirigent son évaluation, quels sont les éléments qu'il prend en compte dès lors qu'il porte un jugement sur la valeur scolaire de ses élèves. Il s'agit de montrer que le jugement scolaire doit être considéré avant tout comme un jugement social qui s'apparente à l'activité évaluative quotidienne que tout un chacun porte sur autrui. Dans cette optique, le jugement a une utilité sociale (Beauvois, 1976, 1984) et doit donc être rapporté au contexte d'interaction (en l'occurrence, la classe) qui ne saurait être indépendant des normes en vigueur dans la société.

Une étude précédente (Bressoux \& Pansu, 1998) a montré que le jugement scolaire est loin de refléter une valeur scolaire "vraie » de l'élève. En effet, si les performances scolaires des élèves jouent un rôle important dans la construction du jugement scolaire de l'enseignant, elles sont loin d'expliquer la totalité de ce jugement. Plusieurs éléments peuvent être invoqués pour rendre compte de ce fait.

Le premier concerne un effet de comparaison intra-classe. Celui-ci se caractérise par le fait que l'évaluation est aussi, et peut-être même avant tout, le résultat d'une comparaison entre les élèves d'une même classe. En ce sens, chaque élève est jugé par rapport à ses pairs : un élève de valeur scolaire donnée pourra être vu comme bon élève dans une classe où beaucoup d'élèves ont un niveau plus faible que le sien, tandis qu'il pourra apparaître comme plutôt faible dans une classe où beaucoup d'élèves ont un 
niveau plus élevé que le sien. De ce point de vue, l'évaluation est un processus de rangement des élèves, lié à une situation de classe particulière.

Le deuxième élément concerne un effet de contexte. Celui-ci indique également un ancrage à une situation particulière, mais dont le mécanisme est différent du précédent. On observe que la distribution des valeurs scolaires attribuées par l'enseignant s'effectue de telle sorte qu'il n'y ait ni trop de notes élevées, ni trop de notes basses, ce qui a pour effet d'homogénéiser les moyennes des différentes classes autour de valeurs qui ne sont elles-mêmes ni très élevées, ni très basses. En conséquence, les enseignants sont globalement d'autant plus sévères que le niveau moyen de leur classe, mesuré par des épreuves standardisées (évaluations nationales de C.E.2) est fort. On peut voir là l'effet d'une norme implicite qui conduirait les enseignants à émettre des jugements socialement acceptables. Un enseignant qui ne donnerait que de bonnes notes, ou qui n'en donnerait que de mauvaises risquerait de n'être guère crédible dans son évaluation aux yeux de ceux qui en auraient connaissance (supérieurs hiérarchiques, collègues, parents, élèves, etc.). En effet, des moyennes de classes très élevées ou très faibles sont $d$ 'autant moins acceptables que les individus n'ont aucun moyen objectif de juger de la valeur des classes les unes par rapport aux autres et que, dès lors, des notes très basses ou très élevées peuvent apparaître comme purement arbitraires. Ce qui remettrait en cause les principes méritocratiques de l'acte évaluatif, et plus généralement de l'École elle-même.

Le troisième élément concerne le fait que des caractéristiques des élèves, autres que leur strict niveau de performances, sont prises en compte par l'enseignant quand il s'agit de porter un jugement sur leur valeur scolaire. Parmi celles-ci figure l'acquisition de la norme sociale d'internalité. Cette norme, caractéristique des sociétés occidentales, « conduit à attribuer de la valeur aux explications (des comportements et des renforcements) qui accentuent le poids causal de l'acteur ", en fait des explications internes (Beauvois, 1994, p. 59; Beauvois \& Dubois, 1988, p. 299; pour revue Dubois, 1994). Les explications causales internes (traits, aptitude, tendance à être...) auraient ainsi plus de valeur que les explications externes (chance, pouvoir d'autrui, situation) parce qu'elles sont normatives et répondent à un besoin social. En effet, il a été à plusieurs reprises postulé que " cette norme sociale d'internalité était associée aux pratiques démocratiques libérales d'exercice du pouvoir " (Beauvois, 1994, p. 60) et qu'elle satisfait des utilités qui garantissent un fonctionnement social (Dubois, 1994). Un pattern d'explications internes serait utile dans la mesure où, indépendamment de l'événement en question, il permet l'évaluation voire plus généralement la gestion des agents sociaux, et permet de dire que « ce que font les gens est le reflet de ce qu'ils sont et doit leur être attribué et que ce qui doit leur arriver (...) est la conséquence de ce qu'ils sont » (Beauvois \& Le Poultier, 1986, p. 100). Parmi les nombreux résultats qui justifient ce point de vue, les plus pertinents pour le propos de cet article, sont ceux qu'apporte le paradigme des juges : une personne ayant fourni des explications internes est quasi systématiquement mieux jugée qu' une personne ayant fourni des explications externes (Jellison \& Green, 1981 ; Beauvois \& Le Poultier, 1986; Dubois, 1988 ; Dubois \& Le Poultier, 1991 ; Dubois, 1994; Castra, 1995; Luminet, 1996; Pansu, 1997; Pansu \& Gilibert, à paraître). Rappelons qu'une étude de Dubois et Le Poultier (1991) confirme l'intervention de la norme d'internalité dans le jugement scolaire des enseignants. Dans celle-ci, des enseignants de C.M.2 devaient énoncer un pronostic concernant le passage en sixième d'écoliers fictifs. Les enseignants disposaient pour ce faire des résultats scolaires desdits élèves (faibles vs moyen faibles), du niveau socio- 
économique de la famille (bas vs élevé) et des réponses des élèves à un questionnaire d'internalité (interne vs externe). Si les résultats montrent comme attendu un effet des résultats scolaires, on constate aussi un effet des réponses au questionnaire d'internalité : les élèves présentés comme internes ont suscité chez les enseignants des jugements plus favorables pour le passage en sixième que les externes. Néanmoins, si de tels résultats tendent à prouver que l'internalité intervient bien dans la construction du jugement évaluatif des enseignants, la situation d'évaluation reste ici artificielle. Les jugements recueillis reflètent donc plus une attitude supposée de l'évaluateur, que le comportement habituel de ce dernier. C'est une des raisons pour lesquelles nous avons voulu étudier dans une première recherche la construction du jugement scolaire en milieu naturel (Bressoux \& Pansu, 1998). Les jugements scolaires étudiés étaient ceux émis par des enseignants à l'égard de leurs propres élèves. Bien que l'internalité des élèves ait été appréhendée par le biais d'un questionnaire d'internalité, il est important de noter que les enseignants n'avaient pas connaissance des réponses des élèves à ce questionnaire. Cela nous permettait d'introduire le facteur internalité dans l'étude tout en évitant de l'imposer expérimentalement comme critère possible d'évaluation aux enseignants, comme on le fait dès lors qu'on se place dans le cadre du paradigme des juges. Les résultats permettent de constater une fois de plus (et ce dans un contexte écologique) que les enseignants privilégient les élèves qui choisissent des explications internes.

\section{Problématique et hypothèses}

Afin de mieux comprendre comment se construit le jugement scolaire qu'un enseignant porte sur ses élèves et, plus particulièrement, de mieux étudier comment intervient la norme d'internalité dans le processus de construction de l'évaluation scolaire, nous avons réalisé une nouvelle étude. Celle-ci a été conçue dans le but de reproduire, en partie, des résultats de même portée théorique que ceux obtenus dans notre première étude (Bressoux \& Pansu, 1998). Il s'agissait de confirmer les résultats relatifs aux effets de contexte et d'envisager quelques extensions susceptibles d'éclairer aux mieux l'impact de la norme d'internalité. Plus précisément, nous testerons tout d'abord une première hypothèse selon laquelle le jugement scolaire du maître se construit, par-delà la performance scolaire des élèves, en fonction de la situation de classe. De plus, eu égard aux travaux sur la norme d'internalité, nous testerons une deuxième hypothèse selon laquelle, toutes choses égales par ailleurs, le jugement scolaire des enseignants est influencé par l'internalité de leurs élèves.

Par ailleurs, dès lors qu'on accepte le point de vue socio-normatif et que, de ce fait, l'internalité n'est plus considérée comme un trait de personnalité (à l'inverse des travaux sur le locus of control, $c f$ Rotter, 1966; pour revue, Dubois, 1987), on peut se demander si l'expression de l'internalité ne varie pas en fonction des sollicitations de l'environnement et de l'image que l'individu veut donner de lui-même. Plus précisément, eu égard au caractère socialement valorisé de l'internalité, on se propose d'étudier si le jugement que portent les enseignants sur la valeur scolaire de leurs élèves varie selon la capacité de ces derniers à se montrer internes lorsqu'il s'agit de se faire bien voir ou externes lorsqu'il s'agit au contraire de se faire mal voir. On rejoint là les travaux sur la clairvoyance normative, lesquels postulent l'intervention d'une variable orthogonale à la normativité et, en particulier, à l'internalité (Py \& Somat, 1991 ; Somat \& Vazel, 1999). Celle-ci relèverait d'une certaine perspicacité vis-à-vis des 
attentes d'autrui et entraînerait en retour une modification des comportements pour s'y adapter. On testera ainsi l'hypothèse selon laquelle les élèves qui savent se montrer internes quand il s'agit de se faire bien voir, ou externes lorsqu'il s'agit de se faire mal voir, feront l'objet d'un jugement plus favorable de la part de l'enseignant. Ils auraient en effet une meilleure capacité à percevoir les demandes de leur environnement et à s'y conformer.

\section{Méthode}

\section{Participants}

L'étude a été conduite auprès de 19 classes de C.E.2, soit 406 élèves (âgés entre 8 et 9 ans) et 19 enseignants. Les classes étaient localisées dans les départements de la Savoie et de l'Isère. Elles font partie de l'agglomération chambérienne ou de l'agglomération grenobloise et sont situées dans des secteurs sociologiquement contrastés. On cherchait de la sorte à varier les contextes de scolarisation. Ainsi, le pourcentage d'enfants d'ouvriers varie, d'une classe à l'autre, de $8 \%$ à $57 \%$ tandis que le pourcentage d'enfants de cadres varie de $0 \%$ à $32 \%$.

\section{Matériel}

Le matériel était constitué d'une fiche individuelle de renseignements concernant les élèves et d'un questionnaire d'internalité présenté selon trois consignes spécifiques.

\section{Fiche individuelle}

Sur la fiche individuelle, plusieurs informations sur les élèves ont été recueillies : d'une part, des informations socio-démographiques telles que le sexe, la profession des parents, le nombre de frères et sœurs et, d'autre part, des informations scolaires telles que le redoublement et les scores obtenus aux épreuves nationales d'évaluation de C.E. 2. Ces épreuves standardisées, qui portent uniquement sur les acquis en français et en mathématiques, sont appréhendées ici comme une base de comparaison des performances scolaires des élèves. Nous ne considérons pas les scores obtenus comme des mesures d'un "vrai » niveau d'acquisitions; leur intérêt est de fournir un étalon de mesure commun à toutes les classes et donc de rendre comparables les niveaux de performance des élèves.

Sur cette fiche, les enseignants devaient noter de 0 (élève très faible) à 10 (élève très fort) chacun de leurs élèves en français et en mathématiques. On dispose ainsi, pour ces deux disciplines, de deux mesures distinctes de la valeur scolaire des élèves : l'une provenant du jugement que l'enseignant porte sur la valeur scolaire de ses élèves et qui constitue l'objet propre de cette étude, l'autre provenant des scores aux évaluations nationales et qui constitue pour nous une variable indépendante.

\section{Questionnaire}

Afin de différencier les élèves du point de vue de leur acquisition de la norme d'intemalité, nous avons construit un questionnaire d'intemalité adapté à des situations scolaires. Ce questionnaire est une version modifiée de celui présenté dans 
Pansu, Bressoux, Leonesio et Mezière (2000). Il comprend 16 items expérimentaux (8 comportements et 8 renforcements) relatant brièvement une situation scolaire suivie de deux types d'explications, l'une interne, l'autre externe, entre lesquelles les élèves doivent choisir ( $c f$ annexe). Par exemple :

Lorsqu'ils ont une bonne note, certains élèves disent : "c'est parce que le maître est gentil". Certains élèves disent : "c'est parce que j'ai bien travaillé" ».

Afin de masquer une dichotomie qui risquait d'être trop apparente dans le choix systématiquement proposé entre une explication interne et une explication externe, nous avons réparti quatre items de remplissage parmi les seize items expérimentaux : ils proposaient tantôt des explications exclusivement internes, tantôt des explications exclusivement externes.

\section{Procédure}

Dans un premier temps, les élèves étaient invités à répondre spontanément à ce questionnaire (consigne standard). Il était dit aux élèves : «je vais te demander de répondre à quelques questions. Ce n'est pas un exercice. C'est juste pour savoir ce que tu penses de certaines choses. Il n'y a pas de bonnes ni de mauvaises réponses. Tes réponses seront toujours onnes si tu dis ce que tu penses réellement ». Après la lecture de chaque item, il était dit : « toi, tu dirais plutôt comment, comme ceux qui disent c'est parce que... (rappeler la première explication) ou comme ceux qui disent c'est parce que... (rappeler la deuxième explication)?».

Le questionnaire a fait l'objet d'une analyse psychométrique. Sa consistance s'est révélée relativement faible (alpha de Cronbach $=0,55)$. Une analyse factorielle a confirmé le faible degré d'homogénéité des items mais n'a pas montré d'items se comportant différemment des autres, ce qui aurait justifié qu'ils soient retirés du calcul du score global d'intemalité. Le score d'intemalité a été construit par sommation des items internes. Si, théoriquement, ce score peut varier de 0 à 16 , dans les faits, il varie de 3 à 16 (moyenne = 10,62 écart-type $=2,51 ;$ cffigure 1).

\section{Figure 1. Distribution des scores d'internalité}

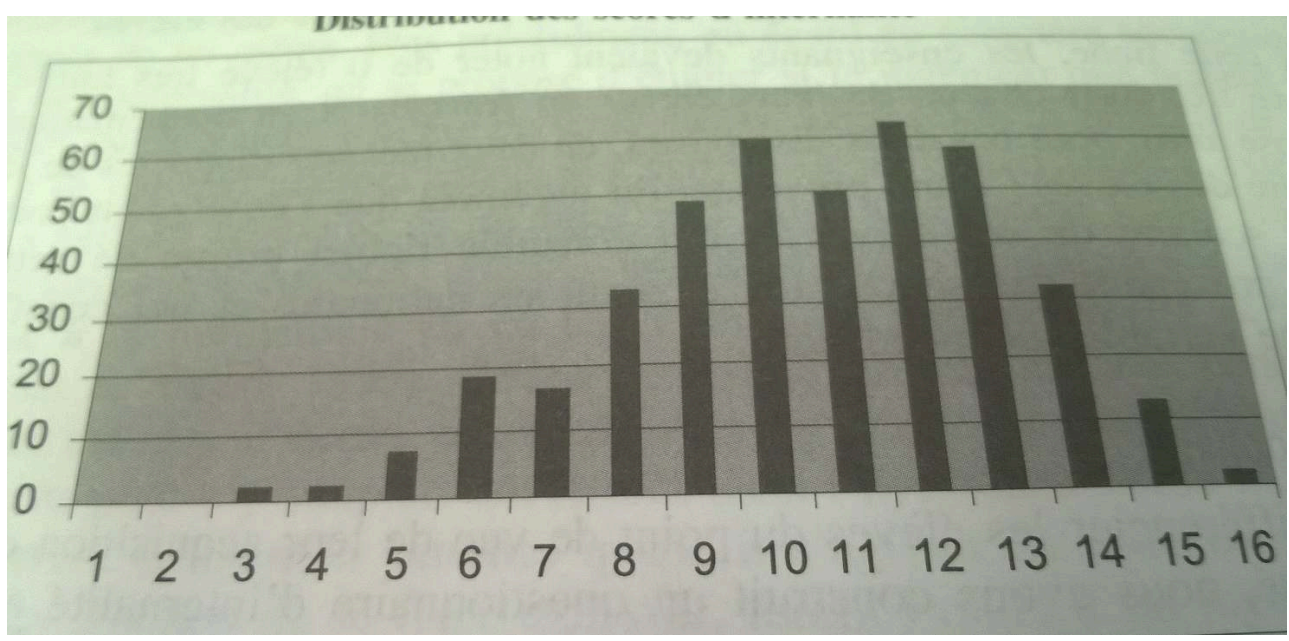

Graph. 1. Distribution of scores of internality 
Dans un second temps, les élèves étaient de nouveau invités à répondre au questionnaire d'intemalité mais, cette fois, avec la consigne de se faire bien voir par le maitre (consigne d'approbation sociale), puis de se faire mal voir par le maître (consigne de désapprobation sociale). Il était dit : «Maintenant, je vais vous redonner le même questionnaire à remplir. Mais cette fois, je vous demande de répondre aux questions en essayant de vous faire bien voir par votre maître(sse) - versus en essayant de vous faire mal voir par votre maitre(sse) - . Comme si vous vouliez que votre maître(sse) soit content(e) de vous - versus ne soit pas content(e) de vous - ». Afin de contrôler un effet d'ordre, les deux dernières consignes ont été contrebalancées.

Ces deux consignes supplémentaires ont été introduites afin de tester la clairvoyance des élèves à l'égard de la valorisation sociale des explications internes.

\section{Analyse statistique}

L'étude réalisée est de type écologique en ce sens qu'elle se déroule en milieu « naturel ». Rappelons que ce qui nous intéresse, c'est le jugement que porte l'enseignant sur ses propres élèves. Celui-ci s'opère dans une situation scolaire particulière, celle de la classe, dont l'effet sur la construction même de ce jugement nous intéresse au premier chef. Il ne s'agit donc pas d'une situation de laboratoire où le chercheur ferait varier un facteur expérimental dont les modalités clairement déterminées l'intéresseraient pour elles-mêmes. Les classes sont bien pour nous une source de variation, mais dont les modalités sont aléatoires : chaque classe n'est pas considérée pour elle-même, mais comme la représentante d'un ensemble plus vaste de classes.

Les données intègrent donc deux types "d'unités » statistiques aléatoires : les élèves et les classes. Les élèves étant intégrés dans les classes, les données présentent de ce fait une structure hiérarchisée, dont le premier niveau est composé par les élèves et le second par les classes.

Il faut donc respecter cette structure hiérarchisée et considérer que chacun de ces deux niveaux est susceptible d'influer sur le phénomène étudié. Cela impose des contraintes tout à fait particulières dans le traitement statistique des données :

D'une part, distinguer les effets propres à chacun de ces niveaux : les techniques « classiques » de modélisation par les moindres carrés ordinaires imposent soit d'agréger les unités de niveau 1 au niveau 2, soit de "désagréger » les unités de niveau 2 au niveau 1. Dans tous les cas, cela revient à écraser la structure hiérarchisée des données puisqu'on ne prend en compte qu'un seul niveau de variation : soit celui des classes, soit celui des élèves ${ }^{1}$.

D'autre part, considérer ces effets comme aléatoires : cela impose que les modalités d'une variable (ici, les différentes classes étudiées) soient traitées comme des réalisations d'un nombre infini de modalités. C'est la distribution des effets de cette infinité de modalités qu'on cherche à estimer, et non l'effet particulier de chacune des modalités observées comme c'est le cas dans un modèle de régression « classique », qui estime donc des effets fixes et non des effets aléatoires.

Dans cette optique, l'emploi de modèles multiniveaux s'impose. Ils ont en effet été spécialement conçus pour l'étude de structures hiérarchisées composées de plusieurs niveaux dont les unités sont aléatoires (Bressoux, Coustère, \& LeroyAudouin, 1997). Afin d'expliquer le jugement que l'enseignant porte sur ses élèves, quatre modèles ont ici 
été estimés et ce pour chacune des deux disciplines : le modèle 1 est dit « vide », c'est-àdire un modèle sans variables indépendantes. Il correspond à une analyse de la variance avec effets aléatoires qui décompose la variance totale de la variable dépendante selon les deux niveaux de la structure hiérarchisée : les élèves et les classes ; le modèle 2 inclut comme variables indépendantes le score des élèves aux épreuves nationales d'évaluation de C.E.2 et le score moyen de la classe à ces mêmes épreuves; le modèle 3 inclut, en plus du modèle précédent, des variables socio-démographiques (profession du père, sexe) et scolaires (redoublement $v s$ non-redoublement) ; enfin, le modèle 4 , considéré comme le modèle complet, inclut, en plus du modèle 3 , les variables qui caractérisent l'internalité des élèves en situation standard, en situation d'approbation sociale et en situation de désapprobation sociale. Précisons que ce modèle permet de raisonner « toutes choses égales par ailleurs ", l'effet de chacune des variables incluses dans le modèle étant estimé à la marge des effets des autres variables ; on obtient ainsi l'effet spécifique (ou effet net) de chaque variable.

Par ailleurs, précisons que la clairvoyance ne sera pas mesurée dans cette étude, comme c'est généralement le cas, par une différence entre le score obtenu en consigne d'approbation sociale et celui obtenu en consigne de désapprobation (Py \& Somat, 1991 ; Channouf, Py, \& Somat, 1995). Cette manière de procéder pose en effet, selon nous, quelques problèmes. D'une part, une même différence a-t-elle la même signification entre deux scores élevés (16-14 par exemple) et entre deux scores faibles (4-2 par exemple) ? D'autre part, n'y a-t-il pas un lien entre ces scores et celui obtenu en consigne standard ? Enfin, n'y a-t-il pas un intérêt à prendre en compte spécifiquement la capacité d'un élève à se montrer interne lorsqu'il s'agit de se faire bien voir et sa capacité à se montrer moins interne lorsqu'il s'agit de se faire mal voir ? Tous ces éléments nous ont conduits à inclure dans l'analyse les scores obtenus en consignes standard, d'approbation sociale et de désapprobation sociale. On cherchera, par l'intermédiaire des modèles multivariés, à estimer l'effet de chacun de ces scores sur le jugement de l'enseignant, les autres étant maintenus constants.

\section{Résultats}

\section{Les parts de variance inter et intra-classes du jugement scolaire des enseignants}

Il s'agit dans un premier temps d'estimer, dans la variation totale des jugements formulés par les enseignants, les parts de variance intra et inter-classes qui correspondent aux deux niveaux de la hiérarchie. On peut ainsi estimer l'ampleur des variations qui se situent entre les élèves à l'intérieur des classes et l'ampleur des variations qui se situent entre les classes. Cette estimation est fournie par le coefficient de corrélation intra-classe (rho : rapport de la variance inter sur la variance totale). En français, rho $=0,104$ ce qui signifie que $10,4 \%$ de la variance totale des jugements que portent les enseignants sur la valeur scolaire de leurs élèves en français se situent entre les classes. En mathématiques, rho $=0,087$ ce qui signifie que $8,7 \%$ de la variance totale $\mathrm{du}$ jugement que portent les enseignants sur la valeur scolaire de leurs élèves en mathématiques se situent entre les classes. Autrement dit, même si la majeure partie des différences se situe au sein des classes, il existe des classes où le jugement scolaire est globalement plus élevé que d'autres. 
Cela nous conduit à penser que le milieu classe est susceptible d'influer sur le jugement que l'enseignant porte sur chacun de ses élèves. Il convient toutefois de préciser que ce milieu peut être caractérisé par deux types de variables :

- soit des caractéristiques agrégées du public d'élèves, qui sont obtenues par calcul à partir de caractéristiques qui sont initialement de niveau 1 : la moyenne des scores individuels donne le niveau d'ensemble de la classe, l'écart-type donne l'hétérogénéité de son public...

- soit des variables "globales " (Boudon, 1970) qui relèvent strictement du niveau 2: organisation pédagogique, expérience de l'enseignant, taille de classe, classe à cours simple ou multiple...

Les variables agrégées identifient le groupe d'élèves en tant que tel ; elles caractérisent ce que nous nommons le contexte de la classe. De ce fait, le contexte est une composante du milieu classe, lequel englobe également les caractéristiques globales. Ce qui nous intéresse ici dans l'effet du milieu classe, c'est spécifiquement l'effet de contexte.

Les différents modèles fournissent des indications sur l'évolution des parts de variance intra et inter-classes au fur et à mesure qu'on inclut des variables supplémentaires ( $c f$ tableaux 1 et 2). Tout d'abord, soulignons que c'est le passage du modèle 1 au modèle 2 qui entraîne des réductions importantes dans les parts de variance intra et inter-classes ; le modèle 2, qui inclut le score des élèves aux épreuves nationales d'évaluation de C.E. 2 et le score moyen de la classe à ces mêmes épreuves est donc globalement explicatif du phénomène étudié. En français, il explique environ $60 \%[(3,364-1,350) / 3,364]$ de la variance intra-classe du jugement de l'enseignant et $27 \%[(0,391-0,285) 10,391]$ de la variance inter-classes. En mathématiques, le modèle est moins explicatif : $47 \%$ [(2,767 $-0,262) 12,767]$ de la variance intra-classes et seulement $5 \%[(0,262-0,249) 10,262]$ de la variance inter-classes. Globalement, on voit donc que, au niveau 1, même si le jugement est fortement lié aux performances individuelles des élèves, il ne se confond pas avec elles. Quant au niveau 2, il existe manifestement d'autres facteurs susceptibles de rendre compte des différences globales de jugement entre les classes.

Le passage du modèle 2 aux modèles 3 puis 4 (cf tableaux 1 et 2 ) n'entraîne que des réductions minimes dans les parts de variance résiduelles. C'est dire que, même si certaines variables incluses dans ces modèles exercent des effets statistiquement significatifs, leur pouvoir explicatif est très modeste : au-delà de leur effet moyen, il existe une forte variabilité interindividuelle. On relève toutefois que la réduction des parts de variance résiduelle est un peu plus marquée en mathématiques qu'en français. Les variables supplémentaires incluses dans les modèles 3 et 4 sont donc un peu plus explicatives dans la première discipline que dans la seconde. En effet, lorsqu'on passe du modèle 2 au modèle 4 , on observe :

- en mathématiques : un gain explicatif de 3,8 points de variance inter-classes et de 2,6 points de variance intra-classe ;

-en français : un gain de seulement 2,1 points de variance inter-classes et de 0,9 point de variance intra-classe.

Nous allons maintenant nous intéresser aux coefficients attachés aux différentes variables. Pour cela, nous nous baserons sur les estimations produites par le modèle 4, qui est le plus complet (cf. tableaux I et 2). 
TABLEAU 1. Modèle expliquant le jugement de l'enseignant sur la valeur scolaire des élèves en français

\begin{tabular}{|c|c|c|c|c|}
\hline PARAMÈTRES & $\begin{array}{l}\text { MODÈ1_F } \\
1\end{array}$ & MODÈLE 2 & MODÈLE 3 & MODÈLE 4 \\
\hline \multicolumn{5}{|l|}{ Effets fixes } \\
\hline Constante & $\begin{array}{l}6,235 \\
(0,170)^{* *}\end{array}$ & $\begin{array}{l}-\quad 0,560 \\
(1,854)\end{array}$ & $0,031(1,882)$ & $1,004(2,075)$ \\
\hline $\begin{array}{l}\text { Score individuel en } \\
\text { français }\end{array}$ & & $\begin{array}{l}0,110 \\
(0,004)^{* *}\end{array}$ & $0,106(0,005)^{* *}$ & $0,103(0,005)^{* *}$ \\
\hline $\begin{array}{l}\text { Score moyen de la } \\
\text { classe en français }\end{array}$ & & $\begin{array}{l}-\quad 0,042 \\
(0,019)^{*}\end{array}$ & $-0,042(0,019)^{*}$ & $-0,041(0,019)^{*}$ \\
\hline 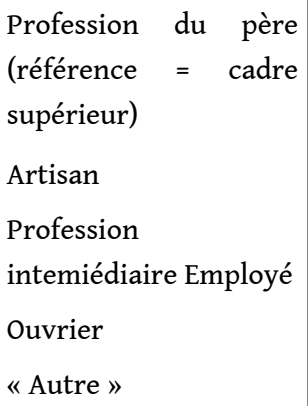 & & & $\begin{array}{llll}- & 0,280 & (0,325) & 0,013 \\
(0,227)- & 0,255 & (0,212) & - \\
0,042(0,214) & - & 0,133 \\
(0,218) & & \end{array}$ & $\begin{array}{lrr}- & 0,291 & (0,323) \\
0,050(0,227) & -0,233 \\
(0,210) & 0,035 & (0,214) \\
0,086 & (0,217) & \end{array}$ \\
\hline Garçon & & & $-0,148(0,119)$ & $-0,156(0,120)$ \\
\hline Redoublement & & & $-0,323(0,172)$ & $-0,352(0,172)^{*}$ \\
\hline Score int. standard & & & & $-0,325(0,158)^{*}$ \\
\hline $\begin{array}{l}\text { Score int. standard } \\
\text { quadratique }\end{array}$ & & & & $0,015(0,008)^{*}$ \\
\hline $\begin{array}{l}\text { Score int. en } \\
\text { approbation sociale }\end{array}$ & & & & $0,077(0,029)^{* *}$ \\
\hline $\begin{array}{l}\text { Score int. en } \\
\text { désapprobation sociale }\end{array}$ & & & & $0,005(0,023)$ \\
\hline Effets aléatoires & & & & \\
\hline Variance inter-classes & $\begin{array}{l}0,391 \\
(0,190)^{*}\end{array}$ & $\begin{array}{l}0,285 \\
(0,125)^{*}\end{array}$ & $0,280(0,123)^{*}$ & $0,277(0,122)^{*}$ \\
\hline Variance intra-classe & $\begin{array}{l}3,364 \\
(0,236)^{* *}\end{array}$ & $\begin{array}{l}1,350 \\
(0,097)^{* *}\end{array}$ & $1,347(0,098)^{* *}$ & $1,320(0,096)^{* *}$ \\
\hline $\begin{array}{l}\text { Part de variance inter- } \\
\text { classes expliquée }\end{array}$ & & $27,1 \%$ & $28,4 \%$ & $29,2 \%$ \\
\hline
\end{tabular}


Part de variance intra-

classe expliquée

\begin{tabular}{|l|l}
$559,9 \%$ & $60,0 \%$
\end{tabular}

$60,8 \%$

(note : Score int. = score d'internai té)

$N=406$

Seuil de significativité :

$p<0,05$ *

$\mathrm{p}<0,01 * *$

TABLE 1. Pattern explaining the school assessment in French

TABLEAU 2. Modèle expliquant le jugement de l'enseignant sur la valeur scolaire des élèves en mathématiques

\begin{tabular}{|c|c|c|c|c|}
\hline PARAMÈTRES & MODÈLE I & MODÈLE 2 & MODÈLE 3 & MODÈLE 4 \\
\hline \multicolumn{5}{|l|}{ Effets fixes } \\
\hline Constante & $\begin{array}{l}6,681 \\
(0,143)^{* *}\end{array}$ & $\begin{array}{l}2,301 \\
(1,677)\end{array}$ & $3,164(1,700)$ & $4,687(1,930)^{*}$ \\
\hline $\begin{array}{l}\text { Score individuel en } \\
\text { mathématiques }\end{array}$ & & $\begin{array}{l}0,089 \\
(0,005)^{* *}\end{array}$ & $0,085(0,005)^{* *}$ & $0,082(0,005)^{* *}$ \\
\hline 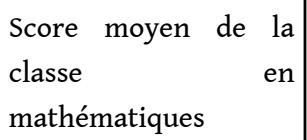 & & $\begin{array}{l}-\quad 0,046 \\
(0,017)^{*}\end{array}$ & $-0,048(0,017)^{*}$ & $-0,046(0,017)^{*}$ \\
\hline $\begin{array}{l}\text { Profession du père } \\
\text { (référence = cadre } \\
\text { supérieur) Artisan } \\
\text { Profession } \\
\text { intermédiaire } \\
\text { Employé } \\
\text { Ouvrier } \\
\text { ex Autre» }\end{array}$ & & & $\begin{array}{llll}-0,017 & (0,343) & - & 0,020 \\
(0,235) & - & 0,265 & (0,219) \\
0,508 & (0,221)^{*} & - & 0,183 \\
(0,225) & & & \end{array}$ & $\begin{array}{l}-0,155(0,338) \\
0,017 \quad(0,231)-0,245 \\
(0,216)-0,415(0219)^{*} \\
-0,142(0,223)\end{array}$ \\
\hline Garçon & & & $0,156(0,122)$ & $0,161(0,122)$ \\
\hline Redoublement & & & $-0,295(0,174)$ & $-0,299(0,173)$ \\
\hline Score int. standard & & & & $-0,478(0,162)^{* *}$ \\
\hline $\begin{array}{l}\text { Score Mt_ standard } \\
\text { quadratique }\end{array}$ & & & & $0,023(0,008)^{* *}$ \\
\hline $\begin{array}{l}\text { Score int. en } \\
\text { approbation sociale }\end{array}$ & & & & $0,076(0,030)^{*}$ \\
\hline
\end{tabular}




\begin{tabular}{|l|l|l|l|l|}
\hline $\begin{array}{l}\text { Score int. en } \\
\text { désapprobation } \\
\text { sociale }\end{array}$ & & & $0,004(0,024)$ \\
\hline Effets aléatoires & $\begin{array}{l}0,262 \\
(0,134)^{*}\end{array}$ & $\begin{array}{l}0,249 \\
(0,113)^{*}\end{array}$ & $0,243(0,110)^{*}$ & $0,239(0,108)^{*}$ \\
\hline Variance inter-classes & $\begin{array}{l}1,463 \\
(0,105)^{5 *}\end{array}$ & $1,440(0,105)^{* *}$ & $1,392(0,102)^{* *}$ \\
\hline $\begin{array}{l}\text { Variance intra-classe } \\
(0,194)^{* *}\end{array}$ & $5,0 \%$ & $7,3 \%$ & $8,8 \%$ \\
\hline classes expliquée & $\begin{array}{l}5,767 \\
\text { Part de variance inuu- } \\
\text { classe expliquée }\end{array}$ & $47,1 \%$ & $48,0 \%$ & $49,7 \%$ \\
\hline
\end{tabular}

(note Score int. $=$ score d'internalité) $N=404$

Seuil de significativité :

$\mathrm{p}<0,05^{*}$

$\mathrm{p}<0,01 * *$

TABLE 2. PATTERN EXPLAINING THE SCHOOL ASSESSMENT IN MATHEMATICS

L'influence des caractéristiques socio-démographiques et scolaires des élèves Les variables socio-démographiques (profession du père, sexe) et scolaires (redoublement vs nonredoublement) ont ici un statut de variables de contrôle ; elles servent essentiellement à mesurer l'effet des autres variables toutes choses égales par ailleurs. Bien que leurs effets ne nous intéressent pas directement, on peut noter deux effets significatifs. Le premier concerne la profession du père : par rapport aux enfants de cadres supérieurs, les enfants d'ouvriers font l'objet de jugements plus sévères en mathématiques. Le second concerne le redoublement : les redoublants sont jugés plus sévèrement en français que les autres. Néanmoins, il ne faudrait pas focaliser sur des différences disciplinaires car les coefficients, bien que n'étant pas tous significatifs, sont globalement de même signe en français et en mathématiques.

\section{L'influence du niveau scolaire des élèves et l'effet de contexte}

Comme on pouvait s'y attendre, le jugement de l'enseignant est d'autant plus favorable que le score des élèves aux évaluations nationales de C.E.2 est meilleur. Au-delà de cet effet qu'on peut qualifier de trivial, on observe un effet négatif associé au niveau global de la classe. Le jugement que l'enseignant porte sur ses élèves est d'autant plus sévère que le niveau moyen de la classe est élevé. Cet effet montre que le jugement de l'enseignant est dépendant du contexte, c'est-à-dire qu'il se fonde sur la valeur de l'élève dans une classe déterminée. 


\section{L'influence des scores d'internalité}

Le jugement de l'enseignant est influencé par l'internalité des élèves en consigne standard, mais l'effet n'est pas linéaire (cffigures 2 et 3$)^{2}{ }^{2}$. Le jugement du maître est le plus élevé lorsque les élèves choisissent une forte majorité d'explications externes ou, au contraire, quand ils choisissent une forte majorité d'explications internes. Ce sont les élèves qui ont un score d'internalité " moyen » qui sont le plus sévèrement jugés. Toutes choses égales par ailleurs, ils accusent en moyenne, par rapport aux mieux jugés, un déficit d'environ 1 point sur une échelle qui va de 0 à 10 , ce qui n'est pas négligeable. Si les courbes représentatives de la relation entre les scores d'internalité et le jugement de l'enseignant ont globalement la même allure dans les deux disciplines, on observe toutefois

FIGURE 2. Relation entre le score d'internalité et le jugement de l'enseignant en français

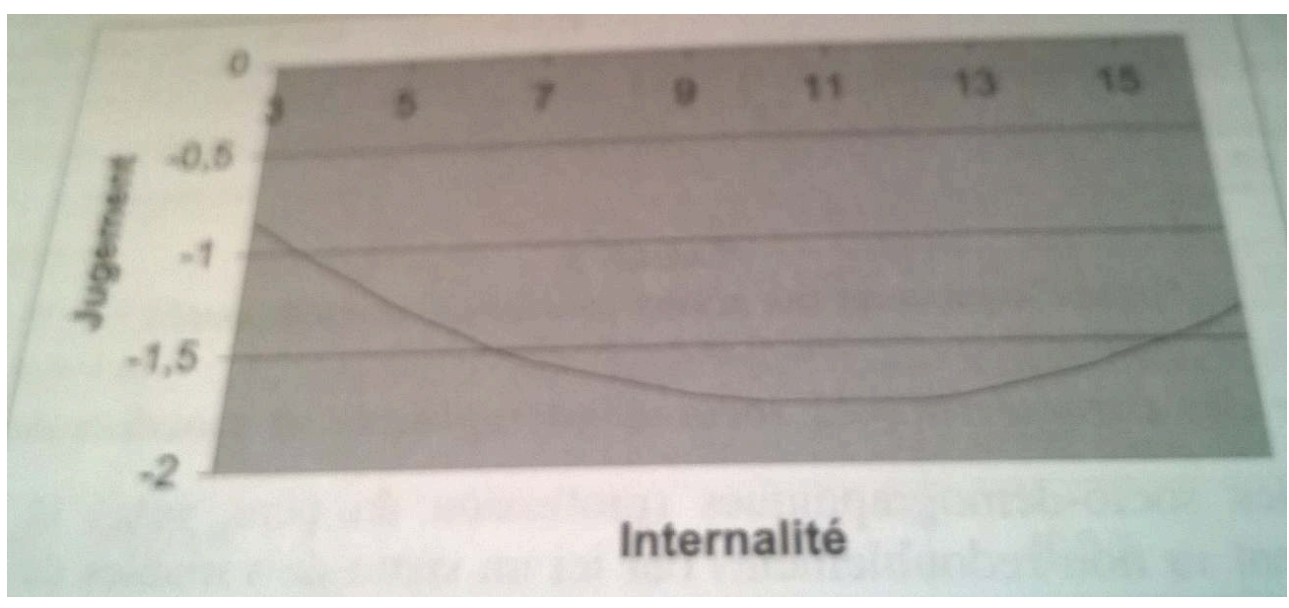

Graph 2. Relation between the score of internality and the school assessment in French

FIGURE 3. Relation entre le score d'internalité et le jugement de l'enseignant en mathématiques

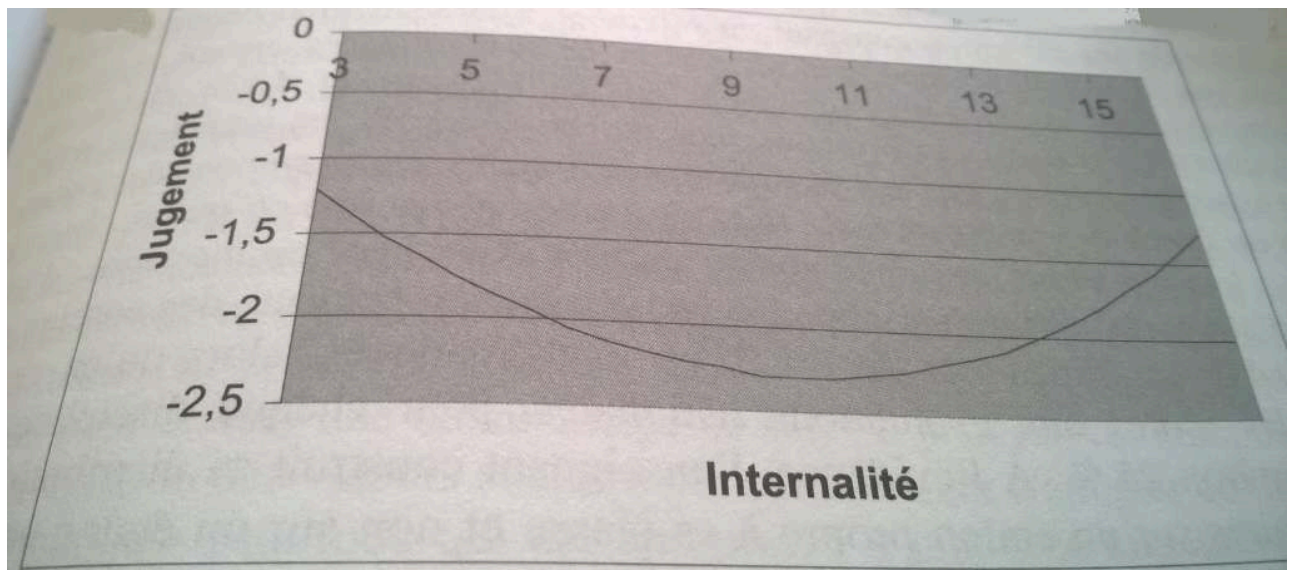

GRAPH 3. Relation between the score of internality and the school assessment in mathematics

une légère différence. La courbe apparaît parfaitement symétrique en mathématiques, mais pas en français. Dans ce dernier cas, on observe que la courbe est plus élevée là où se situent les élèves les plus externes que là où se situent les élèves les plus internes. 
Le jugement de l'enseignant est également influencé par l'internalité des élèves en consigne d'approbation sociale. L'effet est cette fois linéaire et positif : plus le score d'internalité en consigne d'approbation sociale est élevé, meilleur est le jugement de l'enseignant. La capacité des élèves à se montrer internes lorsqu'il s'agit de se faire bien voir semble jouer positivement sur le jugement de l'enseignant.

En revanche, aucun effet significatif n'est observé pour ce qui est du score d'internalité en consigne de désapprobation sociale. Ce résultat indique clairement qu'il n'y a pas de symétrie dans la relation avec le jugement scolaire pour les scores d'internalité obtenus avec les consignes d'approbation et de désapprobation sociales. Cela justifie de prendre en compte séparément chacune de ces deux variables et d'en étudier les effets spécifiques, plutôt que d'intégrer leur seule différence.

\section{Discussion}

L'objectif de cette étude était de montrer que le jugement scolaire doit être considéré avant tout comme un jugement social global qui doit être rapporté au contexte d'interaction (i.e. la classe), qui lui-même ne saurait être indépendant des normes de jugement en vigueur dans la société. Sur ce plan, il s'agissait d'apporter des éléments nouveaux susceptibles de nous éclairer sur l'intervention de la norme d'internalité dans les pratiques d'évaluation scolaire.

Les résultats renforcent ceux obtenus dans une recherche antérieure (Bressoux \& Pansu, 1998). Le jugement que l'enseignant est amené à porter sur la valeur scolaire de ses élèves se construit en référence à une situation déterminée. Il n'est pas la simple traduction d'un niveau de performances en soi mais un processus dans lequel entre également en jeu le contexte de la classe, en particulier le niveau de performances des autres élèves. Si les jugements reflétaient exactement les scores aux évaluations nationales, les moyennes des classes faibles ou fortes devraient être, de fait, plus hétérogènes que ce qu'on observe. En effet, les jugements des enseignants varient assez peu, en moyenne, d'une classe à l'autre ; la part de variance inter-classes des jugements des enseignants est de 10,4 \% en français et de 8,7 \% en mathématiques, alors qu'en ce qui concerne les scores aux évaluations nationales, pour chaque discipline, elle s'élève à environ $25 \%$. À l'évidence, l'enseignant construit sa distribution des valeurs scolaires sur un étalon propre à sa classe et non sur un étalon commun à toutes les classes, comme c'est le cas des évaluations nationales. Cependant, cette construction, qui reflète une situation de classe particulière, s'élabore selon des normes de jugement scolaire, somme toute largement informelles, mais communes aux enseignants. Ces normes peuvent être appréhendées dans l'effet de contexte qui montre que les enseignants tendent à relever le niveau des classes trop faibles et à abaisser celui des classes trop fortes autour de valeurs socialement acceptables. Néanmoins, cet effet de contexte est loin d'expliquer la totalité des différences inter-classes (cf tableaux 1 et 2); cette plus ou moins grande indulgence ou sévérité provient donc également d'autres facteurs parmi lesquels figurent sans doute des caractéristiques propres à l'enseignant telles que celles mises en évidence par Felouzis (1997) dans l'enseignement secondaire (milieu socioculturel, expérience professionnelle, idéologie pédagogique...).

Au-delà de facteurs contextuels, le jugement scolaire s'élabore à partir de caractéristiques propres aux élèves telles que leur acquisition de la norme d'internalité. On voit ici que le jugement des enseignants est le plus élevé lorsque les réponses des 
élèves sont très exclusives : soit une forte majorité d'explications internes, soit une forte majorité d'explications externes. Ces résultats sont en contradiction partielle avec un certain nombre de travaux qui ont montré une relation positive linéaire entre l'internalité des élèves et le jugement de l'enseignant (Bressoux \& Pansu, 1998 ; Dubois, 1994 ; Dubois \& le Poultier, 1991). À ce stade, on peut penser que ce qui compte surtout pour l'enseignant, c'est la cohérence d'un mode explicatif, soit interne, soit externe. De toute évidence ce n'est pas la seule valeur d'internalité, spontanément exprimée, qui est privilégiée ici. On peut faire le lien avec la notion de consistance dont certains ont montré qu'elle était valorisée socialement (Channouf \& Mangard, 1997; Cialdini, 1990; Cialdini, Trost, \& Newsom, 1995) : il existerait une norme de consistance qui se traduirait par la valorisation sociale de la stabilité des attitudes et des comportements, et qui renverrait à une utilité sociale, celle de rendre prévisible le comportement d'autrui. Or, nos instruments ne permettent pas de séparer norme d'internalité et norme de consistance dans la mesure où les élèves très externes ou, au contraire, très internes, apparaissent les uns comme les autres dotés d'une certaine consistance dans leur mode d'explication des événements.

Par ailleurs, la capacité des élèves à se montrer internes lorsqu'il s'agit de donner une bonne image d'eux-mêmes (consigne d'approbation sociale) se révèle, elle, liée de manière positive et linéaire au jugement des enseignants. Ce résultat semble traduire l'effet d'une forme de clairvoyance sur le caractère valorisant des explications internes. C'est peut-être une véritable compétence sociale qui est en jeu ici et qui s'exprime dans l'ajustement d'une attitude à une attente sociale : se montrer interne dès lors qu'il s'agit de se faire bien voir. Cet ajustement ne s'effectue pas nécessairement par une utilisation stratégique de l'internalité (au sens d'une représentation consciente et claire de l'attitude à adopter pour donner une bonne image de soi) ; il peut relever aussi de processus d'intériorisation des valeurs sociales qui conduiraient à adopter de manière quasi " automatique » l'attitude qui est appropriée à une telle situation.

Paradoxalement, l'attitude qu'on pourrait croire symétrique, qui consisterait à se montrer externe pour donner une mauvaise image de soi, ne produit aucun effet significatif sur le jugement de l'enseignant. Cette asymétrie a pu être mise en évidence dans la mesure où nous n'avons pas utilisé le mode de construction classique de la clairvoyance, celui d'une différence entre les scores d'internalité en consigne d'approbation et de désapprobation sociales (Channouf, Py, \& Somat, 1995 ; Py \& Somat, 1991 ; Somat \& Vazel, 1999). Le score obtenu par différence ignore en effet deux situations particulières pour ne retenir que l'écart entre elles. Or, les résultats que nous obtenons montrent l'intérêt qu'il y a à considérer chacune de ces situations comme ayant un intérêt spécifique dans leur effet sur le jugement d'autrui. Lorsqu'on calcule sur nos données un score de clairvoyance par différence, on obtient un effet significatif et positif, mais néanmoins plus faible que celui de l'internalité en consigne d'approbation sociale. Cela montre que le jugement de l'enseignant dépend moins de la clairvoyance au sens classique que d'une clairvoyance au sens d'une capacité à se montrer interne lorsqu'il s'agit de donner une bonne image de soi. De plus, le score de clairvoyance classique n'est pas indépendant des scores d'internalité. Il est bien évidemment fortement corrélé à chacun des scores qui ont servi à le calculer : il est corrélé à $r=0,80(p<0,0001)$ avec le score d'approbation sociale et à $r=-0,86(p<$ $0,0001)$ avec le score de désapprobation sociale. Il est également corrélé avec le score d'internalité en consigne standard $(r=0,41 ; p<0,0001)$. Ce constat va à l'encontre $d u$ postulat d'orthogonalité entre clairvoyance et internalité émis par les chercheurs qui 
étudient la clairvoyance normative. À ce jour, si de tels résultats méritent d'être confirmés, il n'en demeure pas moins qu'ils conduisent à nous interroger sur la notion de clairvoyance, son mode de calcul et sa définition puisqu'elle est ici moins heuristique que l'internalité en consigne d'approbation sociale.

Ces quelques remarques ne sauraient nous faire oublier que cette étude apporte non seulement quelques arguments nouveaux en faveur de la valeur des explications internes mais qu'elle nous éclaire également sur la manière dont le contexte classe affecte le jugement scolaire de l'enseignant. La prise en compte de ces résultats conduit à un certain nombre de conséquences théoriques mais également pratiques. L'évaluation de l'enseignant est en effet primordiale pour le passage dans les classes supérieures et, dès lors, on peut supposer qu'au niveau de performances identiques, le contexte-classe, de même que l'acquisition de la norme d'internalité, influent sur la scolarité des élèves. Ces processus ne jouent toutefois pas avec la même force pour tous les élèves. On l'a vu, le jugement est surtout construit à partir des performances scolaires et les autres variables mises en évidence n'exercent que des effets marginaux. De ce fait, lors des décisions d'orientation, on peut penser que le contexte-classe ou l'internalité ne jouent qu'un rôle mineur quand les élèves ont des niveaux de performances très élevés ou, au contraire, très faibles. Si, pour ces derniers, la décision est avant tout le produit de leurs performances, en revanche, il n'en va sans doute pas de même pour les élèves moyens ou moyens-faibles. Dans ce cas, les effets du contexte de la classe et de la norme d'internalité s'inscrivent dans une marge qui risque bien de faire toute la différence, faisant basculer la décision d'orientation en leur faveur ou en leur défaveur.

\section{BIBLIOGRAPHIE}

Beauvois, J.-L. (1976). Problématique des conduites sociales d'évaluation. Connexions, 19, 7-30. Beauvois, J.-L. (1984). La psychologie quotidienne. Paris : P.U.F.

Beauvois, 1.-L. (1994). Traité de la servitude libérale. Paris : Dunod.

Beauvois, \& Dubois, N. (1988). The norm of internality in the explanation of psychological events. Cahiers de Psychologie Cognitive : European Journal of Social Psychology, 18,

299-316.

Beauvois, J.-L., \& Le Poultier, F. (1986). Norme d'intemalité et pouvoir social en psychologie quotidienne. Psychologie Française, 31, 100-108.

Boudon, R. (1970). L'analyse mathématique des faits sociaux. Paris : Pion.

Bressoux, P., Coustère, P., \& Leroy-Audouin, C. (1997). Les modèles multiniveau dans l'analyse écologique : le cas de la recherche en éducation. Revue française de sociologie, XXX VIII (I), 67-96.

Bressoux, P., \& Pansu, P. (1998). Norme d'intemalité et activités évaluatives en milieu scolaire.

Revue Française de Pédagogie, 122, 19-29.

L'orientation scolaire et professionnelle, 30/3 | 2001 
Castra, D. (1995). Mécanismes implicites de prises de décision dans la situation de recrutement.

L'Orientation Scolaire et Professionnelle, 24, 115-133.

Channouf, A., \& Mangard, C. (1997). Les aspects socionormatifs de la consistance cognitive.

Les Cahiers Internationaux de Psychologie Sociale, 36, 28-45.

Channouf, A., Py, J., \& Somat, A. (1995). Intemalité, clairvoyance normative et pratiques pédagogiques. Les cahiers internationaux de psychologie sociale, 26, 72-87.

Cialdini, R. (1990). Influence et manipulation. Paris : First.

Cialdini, R. Trost, M. R., \& Newsom, J. T. (1995). Preference for consistency : the development of valid measures and the discovery of surprising behavioral implications. Journal of Personality and Social Psychology, 63, 318-328.

Dubois, N. (1987). La psychologie du contrôle : les croyances internes et externes. Grenoble : P.U.G.

Dubois, N. (1988). The norm of intemality : social valorization of behavior and reinforcements in young people. Journal of Social Psychology, 128, 331-439.

Dubois, N. (1994). La norme d'internalité et le libéralisme. Grenoble : P.U.G.

Dubois, N., \& Le Poultier, F. (1.991). Internalité et évaluation scolaire. In J.-L. Beauvois, R.-V. Joule, \& J.-M. Monteil (Eds.), Perspectives cognitives et conduites sociales, 3, Quelles cognitions? Quelles conduites? (pp. 153-166). Cousset (Fribourg) : Delval.

Felouzis, G. (1997). L'efficacité des enseignants. Paris : P.U.F.

Jellison, J. M., \& Green, J. (1981). A self-presentation approach tu the fundamental attribution error : the norm of internality. Journal of Personality and Social Psychology, 40, 643-649.

Luminet, O. (1996). La norme d'internalité dans la consultance en recrutement : variations et clairvoyance dans l'emploi des critères attributifs. Revue Internationale de Psychologie Sociale, 9 , 69-90.

Pansu, P. (1997). Norme d'intemalité et appréciation de la valeur professionnelle : l'effet des

explications internes dans l'appréciation du personnel. Le Travail Humain, 60, 205-222.

Pansu, P., \& Gilibert, D. (sous presse). Effect of causal explanations on work-related judgement.

Applied Psychology: An International Review.

Pansu, P., Bressoux, P, Leonesio, A.-M., \& Mezière, C. (2000). Pour une analyse de la construction du jugement scolaire. Psychologie et éducation, 42, 51-66.

Pieron, H. (1963). Examens et docimologie. Paris : P.U.F.

Py, J., \& Sornat, A. (1991). Normativité, conformité et clairvoyance : leurs effets sur le jugement évaluatif dans un contexte scolaire. In J.-L. Beauvois, R.-V. Joule, \& J.-M. Monteil (Éds.), Perspectives cognitives et conduites sociales 3 Quelles cognitions? Quelles conduites? (pp. 167-193). Cousset (Fribourg) : Delval.

Rotter, J. B. (1966). Generalised expectancies for internai versus external control of reinforcement. Psychological Monographs, 80 (I), Whole n" 609.

Soinat, A., \& Vazel, M. A. (1999). Normative clearsightedness : a general knowledge of social valuation. European Journal of Social Psychology, 29, 691-705. 


\section{NOTES}

1. Entre l'agrégation pure et la désagrégation pure il existe des modélisations intermédiaires, mais qui posent d'autres problèmes (Bressoux, Cousfère, \& Leroy-Audouin, 1997).

2. Ces figures ont été construites à partir des estimations du modèle 4 (cf. tableaux 1 et 2). L'axe des abscisses représente les scores d'internalité observés (compris entre 3 et 16), tandis que l'axe des ordonnées représente l'impact (Le., l'effet marginal) estimé de ces scores sur le jugement de l'enseignant, les autres variables du modèle étant maintenues constantes.

\section{RÉSUMÉS}

La présente étude examine comment les enseignants de C.E.2 construisent le jugement qu'ils portent sur la valeur scolaire de leurs élèves (8-9 ans). Il était demandé, d'une part, aux enseignants de porter des jugements concernant la valeur scolaire de leurs élèves (en mathématiques et en français) et, d'autre part, aux élèves de répondre à un questionnaire d'intemalité selon trois consignes (spontanée, de manière à se faire bien voir, de manière à se faire mal voir). Les résultats montrent premièrement un effet de contexte : plus le niveau global de la classe est élevé, plus le jugement est sévère. Ce résultat confirme l'idée selon laquelle le jugement que l'enseignant porte sur un élève ne se fonde pas uniquement sur le niveau individuel des performances de cet élève, mais également sur le niveau des autres élèves de la classe. Deuxièmement, les résultats montrent que les jugements des enseignants sont influencés par des valeurs normatives comme l'intemalité. Plus précisément, les jugements des enseignants sont influencés par la connaissance que les élèves ont de la norme d'internalité (i.e., lorsqu'il s'agit de se faire bien voir). Ces résultats nous conduisent à discuter la notion de clairvoyance normative.

The present study examines how teachers of CE2 (US third grade and British Junior 2 classes) assess their pupils (8-9 years old). On the one hand, teachers had to assess the mastering of their pupils in two disciplines : mathematics and French. On the other hand, pupils were asked to reply to a questionnaire according to three different instructions : Io reply spontaneously, in a way $w$ be favourably considered (internai) in a way not $w$ be favourably considered (external). The results show, first, a contextual effect ; the higher the Level of achievement of a class is, the more severely the scholastic work is gracled. These results support the view that such an evaluation is not only based upon individual qualifications of a pupil but is in conjonction with the entire class. Second, the restais show that the teachers' assessments are influenced by standards of references such as the internai pattern. More specifically, teaclzers' evaluations are infiuenced by the pupils' knowledge of this internai pattern: to be lavourably considered). These results lead us to discuss the knowledge of the norm of perspicacity.

\section{INDEX}

Keywords : School Assessment, Contextual Effect, Internal Pattern, Normative Perspicacity Mots-clés : Jugement scolaire, effet de contexte, norme d'internalité, clairvoyance normative 


\section{AUTEURS}

\section{PASCAL BRESSOUX}

est Maître de Conférences en Sciences de l'Éducation à l'Université Pierre Mendès France de Grenoble, membre du Laboratoire de recherche des Sciences de l'Éducation. Contact : Université Pierre Mendès France de Grenoble, Département des Sciences de l'Education, 1251 Avenue Centrale, B.P. 47, 38040 Grenoble Cedex 9. Courriel : Pascal.Bressoux@upmf-grenoble.fr

\section{PASCAL PANSU}

est Maître de Conférences en Psychologie Sociale à l'Université de Savoie Chambéry, membre de l'équipe de recherche du Laboratoire de Psychologie Sociale Grenoble-Chambéry (L.P.S.). Contact : Université de Savoie Chambéry, Département de Psychologie, route du Sergent Revel B.P. 1104, 73000 Chambéry. Courriel : Pascal.Pansu@univ-savoie.fr 